\title{
Dérivées et différences divisées à valeurs entières
}

\author{
par \\ JeAn-LuC Chabert (Paris)
}

\section{Introduction}

1. On connait bien, depuis Newton [21] au moins, les polynômes binomiaux

$$
\left(\begin{array}{l}
X \\
n
\end{array}\right)=\frac{X(X-1) \ldots(X-n+1)}{n !} .
$$

Introduits à propos de l'interpolation linéaire et des différences finies (Gregory [16]), ils constituent une base du $\mathbb{Z}$-module des polynômes à valeurs entières sur $\mathbb{Z}$, c'est-à-dire des polynômes $P$ à coefficients rationnels prenant des valeurs entières sur les entiers. Il s'agit essentiellement de la formule de Gregory-Newton :

$$
P(X)=\sum_{n \geq 0} \Delta^{n} P(0) X(X-1)(X-2) \ldots(X-n+1) / n ! .
$$

2. Plus récemment, Straus [27] a étudié l'ensemble $\mathbf{D}$ des polynômes à valeurs entières ainsi que toutes leurs dérivées et montré que $\mathbf{D}$ est un $\mathbb{Z}$-module libre admettant pour base les polynômes

$$
d_{n}\left(\begin{array}{l}
X \\
n
\end{array}\right) \quad \text { où } d_{n}=\prod_{p} p^{[n / p]} .
$$

De son côté, de Bruijn [14] a caractérisé l'ensemble $\boldsymbol{\Delta}^{1}$ des polynômes $P$ à valeurs entières ainsi que leurs premières différences divisées :

$$
\Delta P_{m}(X)=[P(X+m)-P(X)] / m \quad \text { pour } m \in \mathbb{Z}, m \neq 0 .
$$

L'ensemble $\boldsymbol{\Delta}^{1}$ est un $\mathbb{Z}$-module libre admettant pour base les polynômes

$$
\delta_{n}^{1}\left(\begin{array}{c}
X \\
n
\end{array}\right) \quad \text { où } \delta_{n}^{1}=\operatorname{ppcm}\{1,2, \ldots, n\} .
$$

Carlitz [10] a étendu cette caractérisation à l'ensemble $\boldsymbol{\Delta}^{k}$ des polynômes à valeurs entières ainsi que leurs $k$ premières différences divisées successives; 
il s'agit encore d'un $\mathbb{Z}$-module libre admettant pour base les polynômes

$$
\delta_{n}^{k}\left(\begin{array}{l}
X \\
n
\end{array}\right) \quad \text { où } \delta_{n}^{k}=\operatorname{ppcm}\left\{t_{1} t_{2} \ldots t_{r} \mid r \leq k, t_{1}+t_{2}+\ldots+t_{r} \leq n\right\} .
$$

Pour $k=1$, il s'agit du résultat de de Bruijn et, pour $k=\infty$, on voit que l'ensemble $\boldsymbol{\Delta}$ des polynômes à valeurs entières ainsi que toutes leurs différences divisées coïncide avec l'ensemble D étudié par Straus (cf. aussi $[20])$.

3. Or, dès 1919, Pólya [23] et Ostrowski [22] avaient considéré la notion de polynôme à valeurs entières sur un anneau d'entiers de corps de nombres et l'on pouvait se demander si l'égalité $\mathbf{D}=\boldsymbol{\Delta}$, démontrée par Carlitz pour $\mathbb{Z}$, subsistait pour tout anneau d'entiers. Ayant rencontré les différences divisées à propos de l'étude des fonctions lipschitziennes en analyse $p$-adique, Barsky [1] est amené à donner une réponse affirmative ([1], proposition III.6).

Le contre-exemple simple suivant relatif au corps de nombres $\mathbb{Q}(\sqrt{2})$ laisse penser que la réponse est plus nuancée. En effet, le polynôme

$$
P(X)=X^{2}(X-1)^{2} / 2
$$

est à valeurs entières sur l'anneau des entiers $A=\mathbb{Z}[\sqrt{2}]$, ainsi que toutes ses dérivées puisque $P^{\prime}(X)$ appartient à $A[X]$, alors que la différence divisée

$$
[P(\sqrt{2})-P(0)] / \sqrt{2}=-2+3 \sqrt{2} / 2
$$

n'appartient pas à $A$.C'est pourquoi nous nous proposons de caractériser ici les anneaux d'entiers de corps de nombres pour lesquels l'égalité $\mathbf{D}=\boldsymbol{\Delta}$ a effectivement lieu. A cet effet, nous allons nous placer pour commencer dans un cadre plus général.

\section{Notations et définitions}

4. Pour l'instant $A$ désigne un anneau intègre quelconque de corps des fractions $K$. Notons $\mathbf{B}_{A}$ l'anneau des polynômes à valeurs entières sur $A$ :

$$
\mathbf{B}_{A}=\{P \in K[X] \mid P(A) \subset A\} .
$$

Pour tout entier naturel $k$, notons $\mathbf{D}_{A}^{k}$ l'anneau des polynômes à valeurs entières sur $A$ ainsi que leurs $k$ premières dérivées :

$$
\mathbf{D}_{A}^{k}=\left\{P \in \mathbf{B}_{A} \mid P^{\prime} \in \mathbf{D}_{A}^{k-1}\right\}
$$

et $\boldsymbol{\Delta}_{A}^{k}$ l'anneau des polynômes à valeurs entières sur $A$ ainsi que leurs $k$ premières différences divisées:

$$
\boldsymbol{\Delta}_{A}^{k}=\left\{P \in \mathbf{B}_{A} \mid \Delta_{h} P \in \boldsymbol{\Delta}_{A}^{k-1}, h \in A-\{0\}\right\}
$$


où

$$
\Delta_{h} P(X)=[P(X+h)-P(X)] / h .
$$

Bien sûr, nous convenons que

$$
\mathbf{D}_{A}^{0}=\boldsymbol{\Delta}_{A}^{0}=\mathbf{B}_{A} \text {. }
$$

Considérons enfin l'ensemble $\mathbf{D}_{A}$ des polynômes à valeurs entières sur $A$ ainsi que toutes leurs dérivées :

$$
\mathbf{D}_{A}=\bigcap_{k} \mathbf{D}_{A}^{k},
$$

et l'ensemble des polynômes à valeurs entières ainsi que toutes leurs différences divisées :

$$
\boldsymbol{\Delta}_{A}=\bigcap_{k} \boldsymbol{\Delta}_{A}^{k}
$$

[N'oublions pas que, comme pour les dérivées, les différences divisées d'ordre $n+1$ d'un polynômes de degré $n$ sont toutes nulles.]i

L'étude des anneaux $\mathbf{B}$ est entreprise en particulier dans [4], [8], [11]; celle des anneaux $\mathbf{D}^{k}$ dans [3], [5], [13], [24] et celle des anneaux $\boldsymbol{\Delta}^{k}$ dans [17]-[19], [28].

\section{Les inclusions}

5. Lorsqu'il ne peut y avoir de confusion, nous omettrons la référence à l'anneau de base $A$.

Proposition. Pour tout entier $k$, l'anneau $\boldsymbol{\Delta}^{k}$ est inclus dans l'anneau $\mathbf{D}^{k}$.

Il s'agit d'un résultat de Carlitz [10] pour $A=\mathbb{Z}$ et de Haouat et Grazzini [17] pour un anneau intègre $A$ quelconque. Nous en redonnons la démonstration car il y a une petite erreur dans la formulation de [17] :

Lemme. Si $P$ appartient à $\boldsymbol{\Delta}^{k}(k>0)$, alors $P^{\prime}$ appartient $\grave{a} \boldsymbol{\Delta}^{k-1}$.

Démonstration. Pour tout polynôme $P$ de $K[X]$, on a

$$
P(X+Y)=P(X)+Y P^{\prime}(X)+Y^{2} Q(X, Y) \quad \text { où } Q(X, Y) \in K[X, Y] \text {. }
$$

Soit $h$ un élément non nul de $A$ tel que $h Q(X, Y)$ appartienne à $A[X, Y]$. Alors,

$$
P^{\prime}(X)=[P(X+h)-P(X)] / h-h Q(X, h),
$$

c'est-à-dire,

$$
P^{\prime}(X)=\Delta_{h} P(X)+R(X) \quad \text { où } R(X) \in A[X] .
$$

Par suite, si $P$ appartient à $\boldsymbol{\Delta}^{k}(k>0)$, alors $\Delta_{h} P$ appartient à $\boldsymbol{\Delta}^{k-1}$ et donc $P^{\prime}$ aussi. 
Démonstration de la proposition. Montrons par récurrence sur $k$ que $\boldsymbol{\Delta}^{k}$ est inclus dans $\mathbf{D}^{k}$. Pour $k=0$ cela résulte des définitions. Soit $k>0$ quelconque : si $P$ appartient à $\boldsymbol{\Delta}^{k}$, alors $P^{\prime}$ appartient à $\boldsymbol{\Delta}^{k-1}$ d'après le lemme; par hypothèse de récurrence, $\boldsymbol{\Delta}^{k-1}$ est inclus dans $\mathbf{D}^{k-1}$, donc $P$ appartient à $\mathbf{D}^{k}$.

Corollaire. L'anneau $\boldsymbol{\Delta}$ est inclus dans l'anneau $\mathbf{D}$.

6. On peut résumer la situation de la façon suivante :

$$
\begin{aligned}
A[X] \subset \boldsymbol{\Delta} \subset \ldots \subset \boldsymbol{\Delta}^{k} \subset \boldsymbol{\Delta}^{k-1} \subset \ldots \subset \boldsymbol{\Delta}^{1} \\
\cap \\
\mathbf{D} \subset \ldots \subset \mathbf{D}^{k} \subset \mathbf{D}^{k-1} \subset \ldots \subset \mathbf{D}^{1} \subset \mathbf{B} \subset K[X]
\end{aligned}
$$

Dans le cas de $\mathbb{Z}$, l'égalité entre $\mathbf{D}$ et $\boldsymbol{\Delta}$ mise à part, toutes les inclusions ci-dessus sont strictes :

$\mathbb{Z}[X] \neq \boldsymbol{\Delta}$ car $X^{2}(X-1)^{2} / 2 \in \mathbf{D}-\mathbb{Z}[X]$ et $\mathbf{D}=\boldsymbol{\Delta}$.

$\mathbf{B} \neq K[X]$ est évident et $\mathbf{D}^{1} \neq \mathbf{B}$ car $X(X-1) / 2 \in \mathbf{B}-\mathbf{D}^{1}$.

$\boldsymbol{\Delta}^{k} \neq \boldsymbol{\Delta}^{k+1}$ peut se vérifier en utilisant la caractérisation des éléments de $\boldsymbol{\Delta}^{k}$ donnée par Carlitz (cf. $\S 2$ ); ainsi, pour tout nombre premier $p$, la puissance de $p$ qui divise $\delta_{p^{3}}^{k}$ pour $k=p$ est $p^{2 p}$ et pour $k=p^{2}$ est $p^{p^{2}}$.

$\mathbf{D}^{1} \not \subset \boldsymbol{\Delta}^{1}$ car, si $g(X)=\left[X^{p}-X\right] / p$, alors $g_{1}(X)=g(X)^{p} \in \mathbf{D}^{1}$, tandis que $\Delta_{p} g_{1}(0)=\left(p^{p-1}-1\right)^{p} / p \notin \mathbb{Z}$; et même $\mathbf{D}^{k} \not \subset \boldsymbol{\Delta}^{1}$, pour tout $k \geq 1$, car $g_{k}(X)=g(X)^{p k} \in \mathbf{D}^{k}$, tandis que $\Delta_{p} g_{k}(0) \notin \mathbb{Z}$; a fortiori $\mathbf{D}^{k} \not \subset \boldsymbol{\Delta}^{k}$ pour tout $k \geq 1$.

$\mathbf{D}^{k} \neq \mathbf{D}^{k+1}$, sinon $\mathbf{D}^{k}=\mathbf{D}^{k+1}=\mathbf{D}^{k+2}=\ldots=\mathbf{D}=\boldsymbol{\Delta} \subset \boldsymbol{\Delta}^{1}$; ou encore, $p$ désignant un nombre premier au moins égal à $k+2$ :

$h(X)=[X(X-1) \ldots(X-p+1)]^{k+1} / p \in \mathbf{D}^{k}-\mathbf{D}^{k+1} \quad$ (exemple de [2]) .

Dans le cas d'un anneau $A$ d'entiers de corps de nombres on verra que, mise à part l'éventuelle égalité entre $\mathbf{D}$ et $\boldsymbol{\Delta}$ que l'on sait complètement caractériser, toutes ces inclusions sont encore strictes (cf. §15).

\section{Localisation}

7. Soit $S$ une partie multiplicative de $A$. On sait [8] que tout polynôme à valeurs entières sur $A$ est encore à valeurs entières sur $S^{-1} A$, autrement dit

$$
S^{-1}\left(\mathbf{B}_{A}\right) \subset \mathbf{B}_{S^{-1} A} \text {. }
$$

Par suite :

Proposition. Pour toute partie multiplicative $S$ de $A$, on a

$$
S^{-1}\left(\mathbf{R}_{A}\right) \subset \mathbf{R}_{S^{-1} A}
$$

où $\mathbf{R}$ désigne indifféremment l'un des anneaux : $\mathbf{B}, \boldsymbol{\Delta}^{k}, \mathbf{D}^{k}, \boldsymbol{\Delta}$ ou $\mathbf{D}$. 
Démonstration. La vérification des inclusions concernant les anneaux $\mathbf{D}$ est immédiate compte tenu du rappel précédent; celle concernant les anneaux $\boldsymbol{\Delta}$ nécessite l'utilisation répétée de cette inclusion initiale (cf. Haouat et Grazzini [19]). Le principe est le suivant : soit $P$ dans $\boldsymbol{\Delta}_{A}^{1}$; alors, pour tout $h \in A-\{0\}, \boldsymbol{\Delta}_{h} P$ appartient à $\mathbf{B}_{A}$, donc à $\mathbf{B}_{S^{-1} A}$. Fixons $x$ dans $S^{-1} A$ et considérons le polynôme $Q_{x}(Y)=[P(x+Y)-P(x)] / Y=\Delta_{Y} P(x)$. Pour $h \in A-\{0\}, Q_{x}(h)$ appartient à $S^{-1} A$, donc $Q_{x}(A)$ est inclus dans $S^{-1} A$ et $Q_{x}\left(S^{-1} A\right)$ aussi, c'est-à-dire $\Delta_{h} P(x)$ appartient à $S^{-1} A$ pour tout $x$ dans $S^{-1} A$ et pour tout $h$ dans $S^{-1} A-\{0\}$.

On vérifie sans peine les égalités

$$
\mathbf{R}_{A}=\bigcap_{\mathfrak{m} \in \operatorname{Max}(A)} \mathbf{R}_{A_{\mathfrak{w}}}
$$

où, là encore, $\mathbf{R}$ désigne indifféremment : $\mathbf{B}, \boldsymbol{\Delta}^{k}, \mathbf{D}^{k}, \boldsymbol{\Delta}$ ou $\mathbf{D}$. D'où :

Corollaire. Pour que l'égalité $\mathbf{D}_{A}=\boldsymbol{\Delta}_{A}$ ait lieu, il suffit que, pour tout idéal maximal $\mathfrak{m}$ de $A, \mathbf{D}_{A_{\mathfrak{m}}}=\boldsymbol{\Delta}_{A_{\mathfrak{m}}}$.

Cependant cette condition n'est pas toujours nécessaire. Considérons le cas de l'anneau $A$ de tous les entiers algébriques : $\mathbf{B}_{A}=A[X]$ et donc $\mathbf{D}_{A}=\boldsymbol{\Delta}_{A}=A[X]$, tandis qu'il existe des idéaux maximaux $\mathfrak{m}$ de $A$ de corps résiduel fini, au-dessus d'un nombre premier $p$ et dont l'indice de ramification absolu est supérieur à $p$. Donc, compte tenu du $\S 13$ ci-dessous, on a $\mathbf{D}_{A_{\mathfrak{m}}} \neq \boldsymbol{\Delta}_{A_{\mathfrak{m}}}$ pour les localisés $A_{\mathfrak{m}}$ correspondants.

8. Par contre, si l'anneau $A$ est supposé noethérien, les inclusions de la proposition précédente deviennent des égalités :

Proposition. Si l'anneau A est noethérien, alors, pour toute partie multiplicative $S$ de $A$, on a

$$
S^{-1}\left(\mathbf{R}_{A}\right)=\mathbf{R}_{S^{-1} A}
$$

où $\mathbf{R}$ désigne indifféremment l'un des anneaux : $\mathbf{B}, \boldsymbol{\Delta}^{k}, \mathbf{D}^{k}, \boldsymbol{\Delta}$ ou $\mathbf{D}$.

Démonstration. Pour les anneaux $\mathbf{D}$, la preuve est analogue à celle de $\mathbf{B}$ : si $P$ appartient à $\mathbf{D}_{S^{-1} A}^{k}$, c'est-à-dire si $P, P^{\prime}, \ldots, P^{(k)}$ appartiennent à $\mathbf{B}_{S^{-1} A}$, alors il existe $s$ dans $S$ tels que $s P, s P^{\prime}, \ldots, s P^{(k)}$ appartiennent à $\mathbf{B}_{A}$, c'est-à-dire $s P$ appartient à $\mathbf{D}_{A}^{k}$; en effet, le $A$-module engendré par les valeurs de $P, P^{\prime}, \ldots, P^{(k)}$ sur $A$ étant contenu dans le $A$-module de type fini engendré par les coefficients de $P, P^{\prime}, \ldots, P^{(k)}$ est lui-même de type fini. Ce raisonnement s'étend à $\mathbf{D}$ puisqu'un polynôme $P$ n'a qu'un nombre fini de dérivées non nulles. 
Pour les anneaux $\boldsymbol{\Delta}$, indiquons la démonstration dans le cas de $\boldsymbol{\Delta}^{1}$. Pour tout polynôme $P$, posons

$$
P(X+Y)=P(X)+Y P^{\prime}(X)+Y^{2} P^{[2]}(X)+\ldots+Y^{d} P^{[d]}(X)
$$

et soit $N$ le $A$-module de type fini engendré par les coefficients de $P, P^{\prime}$, $P^{[2]}, \ldots, P^{[d]}$. Si $P$ appartient à $\boldsymbol{\Delta}_{S^{-1}}^{1} A$, alors, pour tout $h$ dans $A-\{0\}$ et pour tout $x$ dans $A$, les $\Delta_{h} P(x)=[P(x+h)-P(x)] / h=P^{\prime}(x)+h P^{[2]}(x)+$ $\ldots+h^{d-1} P^{[d]}(x)$ appartiennent au $A$-module de type fini $N \cap S^{-1} A$. Soit $s$ dans $S$ tel que $s\left(N \cap S^{-1} A\right)$ soit inclus dans $A$; alors $s P$ appartient à $\boldsymbol{\Delta}_{A}^{1}$.

Corollaire. Lorsque $A$ est noethérien, pour que $\mathbf{D}_{A}=\boldsymbol{\Delta}_{A}$ il faut et il suffit que, pour tout idéal maximal $\mathfrak{m}$ de $A, \mathbf{D}_{A_{\mathfrak{m}}}=\boldsymbol{\Delta}_{A_{\mathfrak{m}}}$.

\section{Etude locale}

9. Commençons par évacuer les cas simples où $\mathbf{B}=A[X]$ et où donc a fortiori $\mathbf{D}_{A}=\boldsymbol{\Delta}_{A}$. Dans le cas contraire où $\mathbf{B} \neq A[X]$, un certain nombre des inclusions considérées sont par contre toujours strictes pour les "petites valeurs" de $k$ :

Proposition. Supposons l'anneau intègre A noethérien, local, d'idéal maximal $\mathfrak{m}$.

(i) Si A/m est fini (de caractéristique p) et si $\mathfrak{m} \in \operatorname{Ass}(K / A)$, alors $\mathbf{D} \neq A[X]$ et, pour tout $k$ tel que $1 \leq k<p$, on a

$$
\boldsymbol{\Delta}^{k-1} \not \subset \mathbf{D}^{k} \quad \text { et, a fortiori, } \quad \boldsymbol{\Delta}^{k-1} \neq \boldsymbol{\Delta}^{k} \text { et } \mathbf{D}^{k-1} \neq \mathbf{D}^{k} .
$$

(ii) Sinon, $\mathbf{B}=A[X]$ et donc, pour tout $k$,

$$
\boldsymbol{\Delta}=\mathbf{D}=\boldsymbol{\Delta}^{k}=\mathbf{D}^{k}=A[X] .
$$

L'assertion (ii) résulte du :

RAPPEL. Supposons $A$ local d'idéal maximal $\mathfrak{m}$.

(i) Si $A / \mathfrak{m}$ est infini, alors $\mathbf{B}=A[X][8]$.

(ii) Si $A$ est noethérien et si $A / \mathfrak{m}$ est fini, alors $\mathbf{B}=A[X]$ si et seulement si $\operatorname{prof}(K / A)>0$, c'est-à-dire $A=\bigcap_{\mathfrak{p} \neq \mathfrak{m}} A_{\mathfrak{p}}$ [12].

Démonstration de (i). Soit $\alpha$ un élément de $K \backslash A$ tel que $\mathfrak{m} \alpha \subset$ $A$. Posons $g_{k}(X)=\alpha\left(X^{q}-X\right)^{k}$. Nous allons montrer que, pour $1 \leq$ $k<p, g_{k}(X)$ appartient à $\boldsymbol{\Delta}^{k-1}$ et n'appartient pas à $\mathbf{D}^{k}$; de plus, $g_{p}(X)$ appartient à $\mathbf{D}$ et n'appartient pas à $A[X]$.

Posons $s(X)=X^{q}-X$ et vérifions que les polynômes différences divisées d'ordre $\leq k-1$ de $s^{k}(X)$ sont tous à valeurs dans $\mathfrak{m}$ sur $A$. 
Soit $h$ dans $A-\{0\}$; alors

$$
\Delta_{h} s^{k}(X)=\Delta_{h} s(X) \sum_{i=0}^{k-1} s^{i}(X+h) s^{k-i-1}(X)
$$

où $\Delta_{h} s(X) \in A[X]$ et, pour $k-1>0$, chaque terme $s^{i}(X+h) s^{k-i-1}(X)$ est à valeurs dans $\mathfrak{m}$.

Soit $l$ dans $A-\{0\}$; alors

$$
\begin{aligned}
& \Delta_{l} s^{i}(X+h) s^{k-i-1}(X) \\
&= s^{i}(X+h+l) \Delta_{l} s^{k-i-1}(X)+s^{k-i-1}(X) \Delta_{l} s^{i}(X+h) \\
&= s^{i}(X+h+l) \Delta_{l} s(X) \sum_{j=0}^{k-i-2} s^{j}(X+l) s^{k-i-j-2}(X) \\
&+s(X)^{k-i-1} \Delta_{l} s(X+h) \sum_{j=0}^{i-1} s^{j}(X+h+l) s^{i-j-1}(X+h)
\end{aligned}
$$

où $\Delta_{l} s(X)$ et $\Delta_{l} s(X+h) \in A[X]$ et les termes du type $s^{i}(X+h+l) s^{j}(X+$ $l) s^{k-i-j-2}(X)$ sont à valeurs dans $\mathfrak{m}$ dès que $i+j+(k-i-j-2)=k-2>0$.

Et ainsi de suite, d'où $g_{k}(X)$ appartient à $\boldsymbol{\Delta}^{k-1}$. Par ailleurs, supposant toujours $1 \leq k<p$, on vérifie par récurrence sur $i$, que pour $0 \leq i \leq k$, $g_{k}^{(i)}(X)=\alpha\left(X^{q}-X\right)^{k-i} r_{i}(X)$ où $r_{i}(X) \in A[X]$ et $r_{i}(y) \notin \mathfrak{m}$ pour $y \in \mathfrak{m}$.

Par suite, $g_{k}^{(i)}(X)$ appartient à $\mathbf{B}$ pour $0 \leq i<k$, tandis que $g_{k}^{(k)}(y)$ n'appartient pas à $A$ dès que $y$ est dans $\mathfrak{m}$. Ainsi, $g_{k}$ appartient à $\mathbf{D}^{k-1}$ (ce que l'on savait puisque $\left.\boldsymbol{\Delta}^{k-1} \subset \mathbf{D}^{k-1}\right)$, mais n'appartient pas à $\mathbf{D}^{k}$.

Enfin, $g_{p}(X)$ appartient à $\mathbf{D}$, puisque $g_{p}^{\prime}(X)$ est dans $A[X]$.

10. Seul le cas (i) de la proposition précédente se présente pour les localisés d'anneaux d'entiers de corps de nombres. Si nous nous limitons à la dimension 1, la situation à considérer est la suivante :

Hypothèses. L'anneau intègre $A$ est noethérien, de dimension 1 , local, d'idéal maximal $\mathfrak{m}$, de corps résiduel fini de cardinal $q=p^{f}$. Notons aussi $A^{\prime}$ la clôture intégrale de $A$ et $A^{*}$ le complété de $A$ pour la topologie $\mathfrak{m}$-adique.

Si les anneaux $\mathbf{D}$ et $\boldsymbol{\Delta}$ coïncident, ils ont le même spectre et en particulier le même nombre d'idéaux premiers au-dessus de $\mathfrak{m}$. On va voir qu'en général les anneaux $\mathbf{D}^{k}$ et $\boldsymbol{\Delta}^{k}$ sont très différents au niveau de leur spectre et en particulier au niveau des idéaux premiers au-dessus de $\mathfrak{m}$.

RAPPELS. Sous les hypothèses ci-dessus: 
(i) Les idéaux premiers de $\mathbf{B}$ au-dessus de $\mathfrak{m}$ sont de la forme $\mathfrak{m}_{x}=$ $\left\{P \in \mathbf{B} \mid P(x) \in \mathfrak{m} A^{*}\right\}$ où $x$ est un élément quelconque de $A^{*}$ [12].

(ii) Lorsque $A$ est unibranche, c'est-à-dire lorsque $A^{\prime}$ a un seul idéal maximal $\mathfrak{m}^{\prime}$, les idéaux $\mathfrak{m}_{x}$ distincts sont en nombre infini (en bijection avec l'adhérence de $A$ dans le complété de $A^{\prime}$ pour la topologie $\mathfrak{m}^{\prime}$-adique [7]).

(iii) Lorsque $A$ n'est pas unibranche, les idéaux $\mathfrak{m}_{x}$ distincts sont en nombre fini (en bijection avec le quotient de $A$ par un idéal [6] et [15]).

(iv) Les idéaux premiers de $\boldsymbol{\Delta}^{k}$ au-dessus de $\mathfrak{m}$ sont de la forme $\mathfrak{m}_{a}\left(\boldsymbol{\Delta}^{k}\right)=$ $\mathfrak{m}_{a} \cap \boldsymbol{\Delta}^{k}=\left\{P \in \boldsymbol{\Delta}^{k} \mid P(a) \in \mathfrak{m}\right\}$ et ceux de $\boldsymbol{\Delta}$ de la forme $\mathfrak{m}_{a}(\boldsymbol{\Delta})=$ $\mathfrak{m}_{a} \cap \boldsymbol{\Delta}=\{P \in \boldsymbol{\Delta} \mid P(a) \in \mathfrak{m}\}$ où $a$ décrit un système de représentants de $A$ modulo $\mathfrak{m}$; leur nombre est exactement $q[19]$.

(v) Pour tout polynôme $Q$ de $\mathbf{B}$ et pour tout entier $k$, il existe un entier $s$ tel que $Q^{s}$ appartienne à $\mathbf{D}^{k}[13]$.

Proposition. Sous les hypothèses ci-dessus, lorsqu'en outre A est unibranche, pour tout entier $k$, l'anneau $\boldsymbol{\Delta}^{k}$ est strictement contenu dans l'anneau $\mathbf{D}^{k}$. Lorsque de plus $A$ est de caractéristique $p$, l'anneau $\boldsymbol{\Delta}$ est strictement contenu dans l'anneau $\mathbf{D}$.

Démonstration. En effet, pour tout entier $k$, l'application $\mathfrak{m}_{x} \rightarrow$ $\mathfrak{m}_{x}\left(\mathbf{D}^{k}\right)=\mathfrak{m}_{x} \cap \mathbf{D}^{k}$ est une bijection entre les idéaux premiers de $\mathbf{B}$ au-dessus de $\mathfrak{m}$ et ceux de $\mathbf{D}^{k}$ au-dessus de $\mathfrak{m}$ (rappel (v)). L'anneau $A$ étant supposé unibranche, $\operatorname{Spec}(\mathbf{B} / \mathfrak{m B})$ est infini (rappel (ii)), donc aussi $\operatorname{Spec}\left(\mathbf{D}^{k} / \mathfrak{m} \mathbf{D}^{k}\right)$, alors que $\operatorname{Spec}\left(\boldsymbol{\Delta}^{k} / \mathfrak{m} \boldsymbol{\Delta}^{k}\right)$ est de cardinal fini $q$ (rappel (iv)).

[Lorsque $A$ n'est pas unibranche, le nombre fini des idéaux $\mathfrak{m}_{x}$ distincts (rappel (iii)) n'étant pas systématiquement connu, la question reste ouverte.]

Lorsque $A$ est unibranche et de caractéristique $p$, alors, pour tout polynôme $Q$ de $\mathbf{B}, Q^{p}$ appartient à $\mathbf{D}$ et le raisonnement précédent fonctionne encore pour $\mathbf{D}$ et $\boldsymbol{\Delta}$.

[Nous allons voir par contre que, si $A$ est de caractéristique nulle, alors $\operatorname{Spec}(\mathbf{D} / \mathfrak{m D})$ est fini (cf. $\S 12)$.]

\section{Cas local de caractéristique 0}

11. Hypothèses. L'anneau intègre noethérien local $A$, de dimension 1 , de corps résiduel fini (de caractéristique $p$ ), est supposé maintenant de caractéristique 0 . Il existe alors un entier $r$ tel que $\mathfrak{m}^{r(p-1)}$ soit contenu dans l'idéal $p A$.

Proposition. Sous les hypothèses ci-dessus, si $\mathfrak{m}^{p-1} \subset p A$, alors $\mathbf{D}=\boldsymbol{\Delta}$.

En effet, pour montrer que $\mathbf{D}$ est contenu dans $\boldsymbol{\Delta}$ il suffit de vérifier que D est stable par les opérateurs $\Delta_{h}$ où $h \in A-\{0\}$; cela résulte du lemme suivant dans le cas où $r=1$. 
Lemme. Quel que soit le polynôme $P$ de $\mathbf{D}$ et quel que soit l'élément non nul $h$ de $\mathfrak{m}^{r}$, le polynôme $\Delta_{h}(P)$ appartient encore à $\mathbf{D}$.

Démonstration. Soient $P$ dans $\mathbf{D}$ et $h$ dans $\mathfrak{m}^{r}$; alors

$$
\Delta_{h}(P(X))=[P(X+h)-P(X)] / h=\sum_{n \geq 1}\left(h^{n-1} / n !\right) P^{(n)}(X)
$$

où les $P^{(n)}(X)$ sont dans $\mathbf{D}$ et les $h^{n-1} / n$ ! dans $A$, car quel que soit $h \in \mathfrak{m}^{r}$ et quel que soit $n \in \mathbb{N}, h^{n-1} / n$ ! appartient à $A$.

En effet, l'élément $h^{n-1}$ appartient à $\mathfrak{m}^{r(n-1)}$, donc à $\mathfrak{m}^{k r(p-1)}$ où $k$ désigne la partie entière de $(n-1) /(p-1)$, donc à $p^{k} A$. Tout entier premier à $p$ étant inversible dans l'anneau local $A$, il suffit de montrer que $k \geq$ $v_{p}(n !)$ où $v_{p}(n !)$ désigne la plus grande puissance de $p$ divisant $n$ !. Cela revient à $(n-1) /(p-1) \geq v_{p}(n !)$, c'est-à-dire $n-1 \geq(p-1) v_{p}(n !)$ ou $n>(p-1) v_{p}(n !)$. Or,

$v_{p}(n !)=[n / p]+\left[n / p^{2}\right]+\left[n / p^{3}\right]+\ldots<n / p+n / p^{2}+n / p^{3}+\ldots=n /(p-1)$.

R e m ar que. Par contre, s'il existe $h$ dans $\mathfrak{m}$ tel que $h^{p-1}$ n'appartienne pas à $p A$ tandis que $\mathfrak{m}^{p}$ est inclus dans $p A$, alors $\mathbf{D} \not \subset \boldsymbol{\Delta}^{1}$ et donc $\mathbf{D} \neq \boldsymbol{\Delta}$.

En effet, $g(X)=\left(X^{q}-X\right)^{p} / p$ appartient à $\mathbf{B}$ et $g^{\prime}(X)$ appartient à $A[X]$ - donc $g(X)$ est dans $\mathbf{D}$-puisque $\mathfrak{m}^{p} \subset p A$. Par ailleurs, $\Delta_{h} g(0)=$ $\left(h^{q-1}-1\right)^{p} h^{p-1} / p$ n'est dans $A$ pour $h$ dans $\mathfrak{m}$ que si $h^{p-1} \in p A$.

Exemple. Soit $A=\mathbb{Z}[\sqrt{d}]$ où $d$ est un entier sans facteurs carrés et soit $\mathfrak{m}$ un idéal premier de $A$ au-dessus de 2 . L'anneau $A_{\mathfrak{m}}$ est noethérien, de dimension 1 , local, de corps résiduel fini de caractéristique $p=2$. On a $\mathfrak{m}^{2} \subset 2 A$, mais $\mathfrak{m} \not \subset 2 A ;$ donc $\mathbf{D}_{A_{\mathfrak{m}}} \neq \Delta_{A_{\mathfrak{m}}}$.

12. Toujours en caractéristique 0 , les idéaux premiers de $\mathbf{D}$ au-dessus de $\mathfrak{m}$ sont en nombre fini. De manière précise :

Proposition. Si $a \equiv b\left(\bmod \mathfrak{m}^{r}\right)$, alors $\mathfrak{m}_{a}(\mathbf{D})=\mathfrak{m}_{b}(\mathbf{D})$ où $\mathfrak{m}_{a}(\mathbf{D})$ désigne l'idéal maximal $\{P \in \mathbf{D} \mid P(a) \in \mathfrak{m}\}$.

En effet, pour $P$ dans $\mathbf{D},[P(b)-P(a)] /(b-a)=\Delta_{b-a}(P(a))$ appartient à $A$ (lemme du $\S 11)$, donc $P(b)-P(a)$ appartient à $\mathfrak{m}^{r}$ et $P \in \mathfrak{m}_{a}(\mathbf{D})$ équivaut à $P \in \mathfrak{m}_{b}(\mathbf{D})$.

Corollaire 1. Lorsque $\mathfrak{m}^{p-1} \subset p A$, on $a: \mathfrak{m}_{a}(\mathbf{D})=\mathfrak{m}_{b}(\mathbf{D})$ si et seulement si $a \equiv b(\bmod \mathfrak{m})$.

En effet, d'une façon générale, si $\mathfrak{m}_{a}(\mathbf{D})=\mathfrak{m}_{b}(\mathbf{D})$ alors nécessairement $a \equiv b(\bmod \mathfrak{m})$, puisque $X-a$ appartient à $\mathfrak{m}_{a}(\mathbf{D})$. La réciproque résulte de la proposition quand $r=1$.

Corollaire 2. Lorsque A est unibranche de caractéristique 0, les anneaux $\mathbf{D}^{k}$ sont tous distincts. 
En effet, si l'on avait $\mathbf{D}^{k}=\mathbf{D}^{k+1}$ pour un certain entier $k, \mathbf{D}$ serait égal à $\mathbf{D}^{k}$, en contradiction avec le fait que $\operatorname{Spec}\left(\mathbf{D}^{k} / \mathfrak{m} \mathbf{D}^{k}\right)$ est infini (cf. $\left.\S 10\right)$, alors que $\operatorname{Spec}(\mathbf{D} / \mathfrak{m D})$ est fini (proposition précédente).

\section{Cas d'un anneau de valuation discrète}

13. Lorsque $A$ est l'anneau d'une valuation discrète $v$ de corps résiduel fini, pour tout $k$, les anneaux $\mathbf{D}^{k}$ et $\boldsymbol{\Delta}^{k}$ sont distincts $(\S 10)$ et, lorsqu'en outre $A$ est de caractéristique 0 , les anneaux $\mathbf{D}^{k}$ et $\mathbf{D}^{k+1}$ sont eux-aussi distincts $(\S 12)$. De plus :

Soit $h(x)=\left[\left(X^{q}-X\right) / t\right]^{p}$, où $t$ désigne l'uniformisante de $v$. Alors $h(X) \in \mathbf{B}$, mais $D_{t} h(0)=[h(t)-h(0)] / t=\left(t^{q-1}-1\right)^{p} / t$ n'est pas dans $A$, donc $h \notin \boldsymbol{\Delta}^{1}$. Si $v(p) \geq p$, alors $h^{\prime}(x)=\left(X^{q}-X\right)^{p-1}\left(q X^{q-1}-1\right) p / t^{p}$ appartient à $A[X]$, donc $h(X)$ appartient à $\mathbf{D}$ et $\mathbf{D} \not \subset \boldsymbol{\Delta}^{1}$, a fortiori $\mathbf{D} \neq \boldsymbol{\Delta}$. D'où :

Proposition. Si A désigne l'anneau d'une valuation discrète v, les anneaux $\mathbf{D}$ et $\boldsymbol{\Delta}$ sont égaux si et seulement si le corps résiduel de $v$ est infini ou si sa caractéristique p vérifie $v(p)<p$.

D é m o n s t r a t i o n. La condition suffisante résulte du $\S 9$ lorsque le corps résiduel est infini et du $\S 11$ sinon, puisqu'alors $A$ est nécessairement de caractéristique 0 . La condition nécessaire résulte du contre-exemple précédent. Lorsque $A$ est de caractéristique $p$, le contre-exemple fonctionne encore, mais l'assertion résulte aussi du $\S 10$.

On notera que cette condition $v(p)<p$ apparaît incidemment dans [2] à propos d'une remarque sur le spectre de $\mathbf{D}$, dans [24] à propos de la difficulté de détermination d'une base du $A$-module $\mathbf{D}$, mais aussi à plusieurs reprises dans [5] en particulier à propos de la non noethérianité de $\mathbf{D}$.

14. Supposons encore que $A$ soit l'anneau d'une valuation discrète $v$ de corps résiduel fini et que sa caractéristique soit nulle. Non seulement l'ensemble des idéaux premiers de $\mathbf{D}$ au-dessus de $\mathfrak{m}$ est fini ( $(12)$, mais il est en bijection avec $A / \mathfrak{m}$ lorsque $v(p) \leq p-1$ (équivalence ( $\beta$ ) ci-dessous), et avec $A / \mathfrak{m}^{2}$ lorsque $p \leq v(p) \leq 2(p-1)$ (implication ( $\left.\delta\right)$ ci-dessous). Par contre, la question du nombre des idéaux premiers de $\mathbf{D}$ au-dessus de $\mathfrak{m}$ pour $v(p) \geq 2 p-1$ reste ouverte.

En effet, la proposition du $\S 12$ se traduit par

$$
[v(p) \leq r(p-1) \text { et } v(a-b) \geq r] \Rightarrow\left[\mathfrak{m}_{a}(\mathbf{D})=\mathfrak{m}_{b}(\mathbf{D})\right],
$$

et son corollaire 1 par

$$
[v(p)<p] \Rightarrow\left[v(a-b) \geq 1 \Leftrightarrow \mathfrak{m}_{a}(\mathbf{D})=\mathfrak{m}_{b}(\mathbf{D})\right] .
$$


Par ailleurs, si $v(p) \geq p$, le polynôme $h(X)$ de l'exemple précédent appartient à $\mathfrak{m}_{0}(\mathbf{D})$, mais non à $\mathfrak{m}_{t}(\mathbf{D})$; donc $v(p) \geq p$ et $v(a-b) \geq 1$ n'impliquent pas $\mathfrak{m}_{a}(\mathbf{D})=\mathfrak{m}_{b}(\mathbf{D})$. Finalement,

$$
[v(p)<p] \Leftrightarrow\left[v(a-b) \geq 1 \Leftrightarrow \mathfrak{m}_{a}(\mathbf{D})=\mathfrak{m}_{b}(\mathbf{D})\right] .
$$

Mais on a aussi

$$
\left[v(p) \geq p\left(q^{s}-1\right) /(q-1) \text { et } \mathfrak{m}_{a}(\mathbf{D})=\mathfrak{m}_{b}(\mathbf{D})\right] \Rightarrow[v(a-b)>s] .
$$

En effet, supposons que $v(a-b) \leq s$ et considérons un système de représentants de $A$ modulo $\mathfrak{m}^{s}, a_{1}, a_{2}, \ldots, a_{q^{s}}$, contenant $a$ mais non $b$. Posons $w(s)=\left(q^{s}-1\right) /(q-1)$ et $g(X)=t^{-w(s)}\left(X-a_{1}\right)\left(X-a_{2}\right) \ldots\left(X-a_{q^{s}}\right)$. Alors $g(X)$ appartient à $\mathbf{B}, g(a)=0$ tandis que $v(g(b))=0$ (cf. [11]). Si $v(p) \geq p w(s)$, alors $\left(g^{p}\right)^{\prime}$ appartient à $A[X], g^{p}$ est dans $\mathbf{D}$ et finalement appartient à $\mathfrak{m}_{a}(\mathbf{D})$, mais non à $\mathfrak{m}_{b}(\mathbf{D})$.

En particulier, pour $s=1$,

$$
\left[v(p) \geq p \text { et } \mathfrak{m}_{a}(\mathbf{D})=\mathfrak{m}_{b}(\mathbf{D})\right] \Rightarrow[v(a-b) \geq 2],
$$

alors que, pour $r=2$,

$$
[v(p) \leq 2(p-1) \text { et } v(a-b) \geq 2] \Rightarrow\left[\mathfrak{m}_{a}(\mathbf{D})=\mathfrak{m}_{b}(\mathbf{D})\right] ;
$$

d'où

$$
[p \leq v(p) \leq 2(p-1)] \Rightarrow\left[v(a-b) \geq 2 \Leftrightarrow \mathfrak{m}_{a}(\mathbf{D})=\mathfrak{m}_{b}(\mathbf{D})\right] .
$$

\section{Globalisation}

15. Les $\S \S 8$ et 13 conduisent au

ThÉorÈme. Soit $A$ un anneau de Dedekind. Les anneaux $\mathbf{D}$ et $\boldsymbol{\Delta}$ sont égaux si et seulement si, pour tout idéal maximal $\mathfrak{m}$ de $A$, ou bien le corps résiduel $A / \mathfrak{m}$ est infini, ou bien sa caractéristique $p$ vérifie $\mathfrak{m}^{p-1} \subset p A_{\mathfrak{m}}$.

Application au cas de l'anneau des entiers d'un corps de nombres :

Proposition. Soit A l'anneau des entiers d'un corps de nombres $K$. Pour que $\mathbf{D}=\boldsymbol{\Delta}$ il faut et il suffit que chaque idéal maximal $\mathfrak{m}$ de A vérifie les conditions équivalentes suivantes (où $p$ désigne le nombre premier audessous de $\mathfrak{m})$ :

(i) l'indice de ramification e $=e(\mathfrak{m} / p)$ de $\mathfrak{m}$ dans l'extension $K / \mathbb{Q}$ est majoré par $p-1$,

(ii) l'exposant $s$ de $\mathfrak{m}$ dans la différente $\mathcal{D}_{A / \mathbb{Z}}$ est majoré par $p-2$. 
Démonstration. La condition (i) est celle du théorème. Il s'agit de montrer l'équivalence de (i) et (ii). On sait ([26], chap. III) que, d'une façon générale, l'exposant $s$ de $\mathfrak{m}$ dans la différente $\mathcal{D}_{A / \mathbb{Z}}$ est toujours au moins égal à $e-1$ et que l'égalité $s=e-1$ a lieu si et seulement si $p$ ne divise pas $e$. Ici, si $e \leq p-1$, alors $p$ ne divise pas $e$ et donc $s=e-1 \leq p-2$; d'où (i) $\Rightarrow$ (ii). Inversement, si $s \leq p-2$, comme $s \geq e-1$, on a $e \leq s+1 \leq p-1$; d'où (ii) $\Rightarrow$ (i).

De plus :

Proposition. Lorsque A est l'anneau des entiers d'un corps de nombres, l'éventuelle égalité entre $\mathbf{D}$ et $\boldsymbol{\Delta}$ mise à part, toutes les inclusions suivantes sont strictes:

$$
\begin{aligned}
A[X] \subset \boldsymbol{\Delta} \subset \ldots \subset \boldsymbol{\Delta}^{k} \subset \boldsymbol{\Delta}^{k-1} \subset \ldots \subset \mathbf{\Delta}^{1} \\
\cap \\
\quad \mathbf{D} \subset \ldots \subset \mathbf{D}^{k} \subset \mathbf{D}^{k-1} \subset \ldots \subset \mathbf{D}^{1} \subset \mathbf{B} \subset K[X]
\end{aligned}
$$

Démonstration. Pour tout entier $k$, l'anneau $\boldsymbol{\Delta}^{k}$ est strictement contenu dans l'anneau $\mathbf{D}^{k}(\S \S 8$ et 10$)$ et l'anneau $\mathbf{D}^{k}$ est lui-même strictement contenu dans l'anneau $\mathbf{D}^{k-1}$ ( $\S \S 8$ et 12 ). Il reste à s'assurer que les anneaux $\boldsymbol{\Delta}^{k-1}$ et $\boldsymbol{\Delta}^{k}$ sont eux-aussi distincts. Or, pour tout $k$, il existe un nombre premier $p>k$ et un idéal maximal $\mathfrak{m}$ de $A$ au-dessus de $p$. Pour le localisé $A_{\mathfrak{m}}$ correspondant, on a $\boldsymbol{\Delta}_{A_{\mathfrak{m}}}^{p-1} \neq \boldsymbol{\Delta}_{A_{\mathfrak{m}}}^{p-2}(\S 9)$, par suite $\boldsymbol{\Delta}_{A}^{p-1} \neq \boldsymbol{\Delta}_{A}^{p-2}(\S 8)$ et donc $\boldsymbol{\Delta}_{A}^{k} \neq \boldsymbol{\Delta}_{A}^{k-1}$.

16. Corps quadratiques. Lorsque $A$ est l'anneau des entiers d'un corps quadratique $K=\mathbb{Q}[\sqrt{d}]$ où $d$ est un entier sans facteurs carrés, l'égalité $\mathbf{D}=\boldsymbol{\Delta}$ a lieu si et seulement si $d \equiv 1(\bmod 4)$.

En effet, puisque $[K / \mathbb{Q}]=2, e=e(\mathfrak{m} / p)=1$ ou 2 et le seul cas à rejeter est celui où $e=2$ et $p=2$. Or, 2 est ramifié si et seulement si $d \equiv 2$ ou $3(\bmod 4)([25]$, chap. $\mathrm{V})$; reste donc le cas où $d \equiv 1(\bmod 4)$ et $A=\mathbb{Z}+(1+\sqrt{d}) / 2 \mathbb{Z}$.

Corps cyclotomiques. Lorsque $A$ est l'anneau des entiers d'un corps cyclotomique $K=\mathbb{Q}[\zeta]$ où $\zeta$ désigne une racine primitive $n$ ième de l'unité, l'égalité $\mathbf{D}=\boldsymbol{\Delta}$ a lieu si et seulement si $n$ est un produit de nombres premiers distincts.

En effet, les nombres premiers $p$ ramifiés dans l'extension $A / \mathbb{Z}$ sont ceux qui divisent $n$ et l'indice de ramification correspondant est égal à $p^{k-1}(p-1)$ où $p^{k}$ désigne la plus grande puissance de $p$ divisant $n$ ([26], chap. IV). Pour que la condition du théorème soit réalisée, il faut et il suffit donc que $k=1$.

Contre-exemples (pour lesquels $\boldsymbol{\Delta}$ est strictement contenu dans $\mathbf{D}$ ).

(i) L'anneau $A$ d'une courbe plane irréductible et non singulière sur un corps fini $\mathbb{F}_{q}$ (cf. $\left.\S 10\right)$. 
(ii) L'anneau $A$ des entiers du corps de nombres $K=\mathbb{Q}[\sqrt[p]{p}]$ où $p$ désigne un nombre premier quelconque.

En effet, si $\xi$ désigne une racine pième de $p$, on a $\xi^{p}=p$ où $p=[K: \mathbb{Q}]$, donc $\mathfrak{m}=\xi A$ est le seul idéal premier de $A$ au-dessus de $p$ et $e(\mathfrak{m} / p)=p$. En particulier, pour $p=2$, on retrouve l'exemple $\mathbb{Z}[\sqrt{2}]$ donné en introduction.

(iii) L'anneau $\mathbb{Z}[i]$ des entiers de Gauss.

En effet, $i=\sqrt[2]{-1}$ et $-1 \equiv 3(\bmod 4)$ (cas quadratique) ou encore $i=\sqrt[4]{1}$ et $4=2^{2}$ (cas cyclotomique!).

(iv) L'anneau $\mathbb{Z}[\sqrt{d}]$ où $d$ est un entier sans facteurs carrés.

En effet, si $d \equiv 2$ ou $3(\bmod 4)$, il s'agit de l'anneau des entiers de $\mathbb{Q}[\sqrt{d}]$. Par contre, lorsque $d \equiv 1(\bmod 4)$, cela résulte de la remarque du $\S 11$ : si $\mathfrak{m}$ désigne un idéal maximal de $A$ au-dessus de $2, \mathbf{D}_{A_{\mathfrak{m}}} \neq \boldsymbol{\Delta}_{A_{\mathfrak{m}}}$.

\section{Bibliographie}

[1] D. Barsky, Fonctions k-lipschitziennes sur un anneau local et polynômes à valeurs entières, Bull. Soc. Math. France 101 (1973), 397-411.

[2] D. Brizolis, Ideals in rings of integer-valued polynomials, J. Reine Angew. Math. 285 (1976), 28-52.

[3] D. Brizolis and E. G. Straus, A basis for the ring of doubly integer-valued polynomials, ibid. 286/287 (1976), 187-195.

[4] P.-J. Cahen, Polynômes à valeurs entières, Canad. J. Math. 24 (1972), 747-754.

[5] —, Polynômes et dérivées à valeurs entières, Ann. Sci. Univ. Clermont-Ferrand Sér. Math. 10 (1975), 25-43.

[6] - Polynômes à valeurs entières sur un anneau non analytiquement irréductible, J. Reine Angew. Math. 418 (1991), 131-137.

[7] - Integer-valued polynomials on a subset, Trans. Amer. Math. Soc., à paraître.

[8] P.-J. Cahen et J.-L. Chabert, Coefficients et valeurs d'un polynôme, Bull. Sci. Math. 95 (1971), 295-304.

[9] P.-J. Cahen et Y. Haouat, Polynômes, dérivées et différences finies divisées à valeurs entières sur un anneau de pseudo-valuation, C. R. Acad. Sci. Paris Sér. I 306 (1988), 581-584.

[10] L. Carlitz, A note on integral-valued polynomials, Indag. Math. Ser. A 62 (1959), 294-299.

[11] J.-L. Chabert, Anneaux de "polynômes à valeurs entières" et anneaux de Fatou, Bull. Soc. Math. France 99 (1971), 273-283.

[12] —, Les idéaux premiers de l'anneau des polynômes à valeurs entières, J. Reine Angew. Math. 293/294 (1977), 275-283.

[13] —, Polynômes à valeurs entières ainsi que leurs dérivées, Ann. Sci. Univ. ClermontFerrand Sér. Math. 18 (1979), 47-64.

[14] N. G. de Bruijn, Some classes of integer-valued functions, Nederl. Akad. Wetensch. Proc. Ser. A 58 (1955), 363-367.

[15] R. Gilmer, W. Heinzer and D. Lantz, The Noetherian property in rings of integer-valued polynomials, Trans. Amer. Math. Soc., à paraître.

[16] J. Gregor y, Lettre à John Collins du 23 novembre 1670, dans : The Correspondence of Isaac Newton, Cambridge Univ. Press, 1959, I, 45-49. 
[17] Y. Haouat et F. Grazzini, Polynômes et différences finies divisées, C. R. Acad. Sci. Paris Sér. A 284 (1977), 1171-1173.

[18] —,-, Différences finies divisées sur un anneau S(2), ibid. 286 (1978), 723-725.

[19] -,-, Polynômes de Barsky, Ann. Sci. Univ. Clermont-Ferrand Sér. Math. 18 (1979), 65-81.

[20] V. Laohakosol et P. Ubolsri, A short note on integral-valued polynomials, Southeast Asian Bull. Math. 4 (1980), 43-47.

[21] I. Newton, Lettre à John Smith du 8 mai 1675, dans : Mathematical Papers, Cambridge Univ. Press, 1967-1976, IV, 14-21.

[22] A. Ostrowski, Über ganzwertige Polynome in algebraischen Zahlkörpern, J. Reine Angew. Math. 149 (1919), 117-124.

[23] G. Pólya, Über ganzwertige Polynome in algebraischen Zahlkörpern, ibid., 97-116.

[24] K. Rogers and E. G. Straus, Infinitely integer-valued polynomials over an algebraic number field, Pacific J. Math. 118 (1985), 507-522.

[25] P. Samuel, Théorie algébrique des nombres, Hermann, Paris 1967.

[26] J.-P. Serre, Corps locaux, Hermann, Paris 1962.

[27] E. G. Straus, On the polynomials whose derivatives have integral values at integers, Proc. Amer. Math. Soc. 2 (1951), 24-27.

[28] C. G. Wagner, Polynomials over $\mathrm{GF}(q, x)$ with integral-valued differences, Arch. Math. (Basel) 27 (1976), 495-501.

76 RUE CHARLOT

75003 PARIS, FRANCE 